\title{
Akimotoite in the Tenham meteorite: Crystal chemistry and high- pressure transformation mechanisms
}

\author{
Tristan Ferroir $^{a,}{ }^{*}$, Pierre Beck ${ }^{b}$, Bertrand Van de Moortèle ${ }^{a}$, Marcel Bohn ${ }^{d}$, Bruno Reynard ${ }^{a}$, \\ Alexandre Simionovici ${ }^{c}$, Ahmed El Goresy ${ }^{\mathrm{e}}$ and Philippe Gillet ${ }^{\mathrm{a}}$
}

\footnotetext{
a Laboratoire des Sciences de la Terre, Université de Lyon, Ecole Normale Supérieure de Lyon, Université Claude Bernard Lyon 1, CNRS 46 Allée d'Italie, 69364 Lyon Cedex 07, France

${ }^{\mathrm{b}}$ Laboratoire de Planétologie de Grenoble, Université Joseph Fourier, BP 53, 38041 Grenoble, France

${ }^{c}$ Laboratoire de Géophysique Interne et Tectonophysique, OSUG, BP 53X, 38041 Grenoble, France

${ }^{\mathrm{d}}$ Microsonde Ouest, IFREMER and CNRS, Centre de Brest, BP70, F-29280 Plouzane, France

${ }^{\mathrm{e}}$ Bayerisches Geoinstitut, Universität Bayreuth, D-95440 Bayreuth, Germany

*: Corresponding author :
}

\begin{abstract}
:
The transformation of pyroxene to its high-pressure polymorph akimotoite $\left(\mathrm{MgSiO}_{3}\right.$ ilmenite type structure) is documented in the shock-induced melt veins of the L6 Tenham chondrite. Four textural relationships between pyroxene and akimotoite are observed in former pyroxene grains entrained in the shear melt vein and in pyroxene grains attached to the wall of the melt vein. In one of the entrained enstatite grains the transformation to akimotoite is partial. One third of the grain is transformed to a polycrystalline aggregate of akimotoite with a scalloped interface with enstatite. Akimotoite $\left(\mathrm{Fe}_{0,4} \mathrm{Mg}_{1,24} \mathrm{Ca}_{0.07} \mathrm{Na}_{0,12} \mathrm{Al}_{0,14}\right)\left(\mathrm{Si}_{1,963} \mathrm{Al}_{0,037}\right) \mathrm{O}_{6}$ is slightly enriched in $\mathrm{Ca}(\mathrm{CaO}=1.74 \%)$, $\mathrm{Al}$ and $\mathrm{Na}$ with respect to enstatite $(\mathrm{CaO}=0.71 \%)$. Narrow bands of polycrystalline akimotoite with three crystallographic orientations and a chemical composition similar to the surrounding enstatite intersect the other part of the grain. In a second grain entrained in the shear melt vein, tablets of akimotoite are interwoven with a pyroxene glass with a lower $\mathrm{Na}$ and Fe content and a higher $\mathrm{Mg}$ content compared to the adjacent akimotoite. This pyroxene glass is probably the product of $(\mathrm{Mg}, \mathrm{Fe}) \mathrm{SiO}_{3}$-perovskite amorphization. Polycrystalline akimotoite is also formed at the expense of enstatite at the vein wall of the shear melt vein. In that case akimotoite is also enriched in $\mathrm{Ca}(\mathrm{CaO}=2.85 \%) \mathrm{Na}(\mathrm{NaO}=1.72 \%)$ and $\mathrm{Al}\left(\mathrm{Al}_{2} \mathrm{O}_{3}=4.14 \%\right)$. All these observations suggest that akimotoite is mainly formed by solid-state transformation of former pyroxenes with subsequent diffusion of calcium, aluminum and sodium from the chondritic melt of the shear melt vein. Finally, the first complete X-ray diffraction pattern of natural akimotoite is presented.
\end{abstract}

Keywords: tenham; chondrite; shock melt vein; akimotoite; perovskite 


\section{Introduction}

Heavily shocked meteorites and particularly chondrites usually contain shock-induced melt veins (SMV) in which numerous high pressure minerals have been encountered ([Binns et al., 1969], [Mason et al., 1968], [Smith and Mason, 1970] and [Putnis and Price, 1979]). These high-pressure polymorphs are usually derived from olivine, pyroxene and plagioclase and can therefore provide an insight into the Earth deep mantle mineralogy and may mimic the phase transformations occurring in a subducting lithosphere. Better constraining the kinetics of these transformations is important to discuss subduction dynamics, deep earthquakes mechanisms and mechanical properties of the subducting lithosphere (Kerschhofer [Kerschhofer et al., 1998], [Liu et al., 1998] and [Mosenfelder et al., 2001]). Recently, with the addition of new constraints on low- to high-pressure polymorph transformation kinetics ([Kerschhofer et al., 2000], [Kubo et al., 2004], [Hogrefe et al., 1994] and [Mosenfelder et al., 2000]), more and more studies have been concentrating on the relationship between the mode of occurrence of these high pressure phases and the duration of the equilibrium peak 
shock pressure. For a shocked chondrite, the timescale of shock duration has been estimated from both mineralogical and diffusional considerations to be up to a few seconds [3, 31, 18].

The Tenham chondrite fell in 1879 in South Georgy, Queensland Australia. It is classified as an L6 ordinary chondrite of shock stage 4-5. Major phases in this meteorite are olivine, orthoenstatite, diopside, plagioclase, the latter partially converted to maskelynite or lingunite, Fe-Ni alloy and troilite. Due to a very intense shock [26], a network of shock induced melt veins are cross-cutting the whole meteorite. In these shock melt veins, natural $\mathrm{MgSiO}_{3}$ ilmenite type structured, akimotoite, was discovered by Tomioka and Fujino (1997) occurring as aggregates and platelets adjacent to enstatite with topotaxial relationships between each other.

Here, we report on different mode of occurrence of akimotoite observed in the Tenham chondrite. We will address the different transformation mechanisms of enstatite to akimotoite and the crystal chemistry of the latter. We also present the first complete X-ray powder diffraction spectra for akimotoite, which we can be used to precisely determine crystal structure parameters.

\section{Materials and Methods}

Reflected light optical microscopy and scanning electron microscopy (SEM) on a JEOL 840 SEM microscope were used to identify and characterize the different akimotoite settings. To identify the mineral phases and clarify the petrographical relationship between akimotoite and their host mineral or glass, Raman point measurements and mapping were performed. Unpolarized Raman spectra were collected using a Jobin-Yvon LabRam HR800 spectrometer. Raman scattering is excited by an argon ion laser tuned at $514.5 \mathrm{~nm}$, and analyzed in backscattering geometry with gratings of $1800 \mathrm{~g} / \mathrm{mm}$ and a visible CCD camera. The laser beam was focused through microscope objectives (x100) down to a 1 micrometer spot on the sample surface and the backscattered light was collected through the same objective. Acquisition times were typically $300 \mathrm{~s}$. The laser power on the sample was kept at $50 \mathrm{~mW}$ to avoid any damages. Two spectra were acquired for each phase in the $200-1500 \mathrm{~cm}^{-1}$ spectral region. We also used Raman mapping for determining the textural relationship between the different phases. Raman maps were acquired with a motorized XY stage, with a micrometric resolution. For each spot, two spectra were collected ( 6 seconds accumulation time) in the spectral range of $600-1100 \mathrm{~cm}^{-1}$ and averaged afterwards. Spot size was approximately $1 \mu \mathrm{m}$ and a spectrum was measured every $0.7 \mu \mathrm{m}$ motor displacement. Each mineral phase was identified using its most intense band $\left(797 \mathrm{~cm}^{-1}\right.$ for akimotoite, the doublet at $1000 \mathrm{~cm}^{-1}$ for pyroxene, the peak at $843 \mathrm{~cm}^{-1}$ for ringwoodite and a broad feature around $600 \mathrm{~cm}^{-1}$ for the sulfides of the shock melt vein. The composite color scale on the map is calculated from the integrated band intensity.

Chemical compositions of the different phases were measured with a CAMECA SX50 electron microprobe. The analyses were performed at an accelerating voltage of $15 \mathrm{kV}$, a probe current of $12 \mathrm{nA}$ and a $1 \mu \mathrm{m}$-sized spot.

Crystallographic mapping was performed using synchrotron X-ray diffraction at ESRF (European Synchrotron Radiation Facility, Grenoble, France) with a $10 \mu \mathrm{m}$ beam size at an energy of $30 \mathrm{keV}$ corresponding to a wavelength of $0.412 \AA$ on ID09 and a $1.5 \mu$ beam size at an energy $12 \mathrm{keV}$ (wavelength $1.033 \AA$ ) on ID22 beam line.

\section{Results}

Figure 1a shows the studied shear melt vein in a thin section of the Tenham chondrite. A general Raman survey shows that akimotoite is a rather common phase observed either in pyroxene grains entrained in the shear melt vein or in pyroxene from the vein margins. Three representative occurrences of akimotoite have been studied in greater details.

Firstly, akimotoite is observed in an angular grain of former enstatite entrained in a shear melt-vein (Figs. 1b and 3a). BSE images show two distinct zones : a light gray one and a dark gray one hatched by brighter lamellae depicting three orientations of unknown crystallographic relationship with the enstatite (Fig. 3b). Point Raman measurements show that the dark gray zone is mainly enstatite (Fig. 2) with Raman peaks at 234, 362, 393, 411, 519, 547 and $935 \mathrm{~cm}^{-1}$ and the two typical doublets at 659-680 and 1009-1024 $\mathrm{cm}^{-1}$. The light grey part and the lamellae intersecting throughout enstatite (Fig. 3a) were identified as akimotoite with typical peak at $796 \mathrm{~cm}^{-1}$ corresponding to the $A_{g}$ mode and additional peaks at $342,407,477,614$ and $672 \mathrm{~cm}^{-1}$ (Fig. 3) [23, 21]. Raman mapping (Fig. 3c) shows a good correlation with the SEM observations. The $672 \mathrm{~cm}^{-1}$ band is too intense to belong to akimotoite alone. It could belong to troilite cristallites or to amorphous pyroxene.. From a textural point of view phase transformation of preexisting enstatite to polycrystalline akimotoite is total in one third of the enstatite and partial in the remaining part. The thin lamellae of akimotoite start from the interface between polycrystalline akimotoite and enstatite and extend towards the untransformed enstatite (Fig. 3b). They display three different orientations : one is perpendicular to the akimotoiteenstatite boundary, one is sub parallel to the interface, and one crosscuts the former. Orientation of akimotoite crystals with respect to enstatite could not be obtained on this particular section, and different transformation mechanism than proposed by Tomioka (2007) [29] may be active. The polycrystalline akimotoite has an embossed aspect while the lamellae appear smoother. Akimotoite-enstatite boundary is scalloped probably reflecting the propagation of a transformation front. The thickness of the akimotoite lamellae is between $0.4-0.9 \mu \mathrm{m}$ with an average value of 0.63 $\mu \mathrm{m}$. The chemical compositions of the different phases inferred from point measurements are listed in Table 1 together with those of akimotoite, enstatite and silicate perovskite reported by [27,28] and [24]. The chemical composition of polycrystalline akimotoite and enstatite exhibits slight differences. Polycrystalline akimotoite has a formula of $\left(\mathrm{Fe}_{0,4} \mathrm{Mg}_{1,24} \mathrm{Ca}_{0,07} \mathrm{Na}_{0,12} \mathrm{Al}_{0,14}\right)\left(\mathrm{Si}_{1,963} \mathrm{Al}_{0,037}\right) \mathrm{O}_{6}$ and has a higher concentration of $\mathrm{Ca}(\mathrm{CaO}=1.74 \%)$ than parental enstatite 
$(\mathrm{CaO}=0.71 \%)$. Sodium and aluminum concentrations are also higher in akimotoite with respect to enstatite. Lamellar akimotoite composition was measured on the thickest lamellae $(0.9 \mu \mathrm{m})$ and has in turn the same chemical composition as the enstatite host. These differences are confirmed at the scale of the whole grain in the chemical map (Fig. 3d) recorded with the same setting as the one used for point measurements. Furthermore, Ca distribution map shows a depletion in calcium in the SMV adjacent to the akimotoite compared to the SMV near the enstatite part. Raman spectra of akimotoite are also slightly modified by the integration into the cell of $\mathrm{Ca}$ and $\mathrm{Al}$ [22]. Major peaks of akimotoite are shifted towards lower wavenumbers $\left(796 \mathrm{~cm}^{-1}\right.$ compared to $\left.799 \mathrm{~cm}^{-1}\right)$ and the presence of a high quantity of aluminum (around 4\%) leads to a broadening of the main band. This is in agreement with the measurement done by Reynard et al.[22] who showed that $\mathrm{Ag}$ mode of $\mathrm{MgSiO}_{3}$-ilmenite broadens with increasing the aluminum content. Using their FWHM value would lead to a 5 wt $\%$ content of $\mathrm{Al}_{2} \mathrm{O}_{3}$, which is in excellent agreement with the microprobe measurement ( $4.18 \mathrm{wt} \%$ ). Nevertheless, the enlargement of this band could also be due to the shock. The dominant crystalline phase in the melt surrounding the entrained enstatite crystal is ringwoodite (typical bands at 794 and $843 \mathrm{~cm}^{-}$ $\left.{ }^{1}\right)$. The observation of ringwoodite together with akimotoite and liquid indicate shock conditions of 25-45 GPa and a temperature of $2500 \mathrm{~K}( \pm 100 \mathrm{~K})$ (Price et al. [19] and Langenhorst et al. [11]). Xie et al. [31] later refined the pressure to be in the range of 21 to $25 \mathrm{GPa}$ according to mineralogical association in the SMV. The same grain described was also studied by X-ray diffraction. The image plates show continuous diffraction rings indicating that the whole sample is polycrystalline. Figure $4 \mathrm{a}$ shows an example of an integrated 1-D X-ray pattern obtained with a $10 \mu \mathrm{m}$ beam. This integrated pattern was successfully indexed with akimotoite, enstatite and ringwoodite using structural parameters described in Horiuchi et al. [7], Angel and Hugh-Jones [1] and Baur et al. [2]. Cell parameters were refined afterwards using the Le Bail algorithm found in the GSAS package (Larson and Von Dreele, 1994). The pattern of polycrystalline akimotoite (chemical composition given in table 1) was indexed in the R-3 space group with cell parameters of a= $4.74 \pm 002 \AA \mathrm{c}=13.55 \pm 002 \AA$ and a cell volume of $263.96 \pm 002 \AA^{3}$ (Table 2 ). The unit cell of akimotoite is slightly smaller than the one reported by [17] $\left(\mathrm{V}=269 \AA^{3}\right)$ but larger $\left(\mathrm{V}=262.54 \AA^{3}\right)$ than pure $\mathrm{MgSiO}_{3}$-ilmenite type structure refined by [7]. This discrepancy is certainly due to the less precise determination of $\mathrm{V}$ by TEM conducted by [27] and/or chemical composition differences such as iron and aluminum content. X-ray microdiffraction $(1 \mu \mathrm{m}$ beam) on the akimotoite part also reveals the presence of pigeonite that could be either spatially mixed with akimotoite or located below it (Fig $4 \mathrm{~b}$ ).

Another mode of occurrence of akimotoite was observed, at the wall of the shock melt vein (Fig 1c). ). In the studied area, about half of an enstatite grain has been partially melted at the contact with the shear melt vein, and yields ferrite or magnetite-type Raman spectra that will not be discussed here. Akimotoite is observed within the non-molten part of the enstatite grain, in a single $20 \mu \mathrm{m}$-wide band in contact with the melting front. Akimotoite located at the vein edge also shows, like in the first observed grain, a rather high concentration in $\mathrm{Ca}(\mathrm{CaO}=2.85 \%), \mathrm{Na}\left(\mathrm{Na}_{2} \mathrm{O}=1.72 \%\right)$ and $\mathrm{Al}$ $\left(\mathrm{Al}_{2} \mathrm{O}_{3}=4.14 \%\right)$. The values found for $\mathrm{CaO}$ are extremely variable ( $\mathrm{CaO}$ can go up to $20 \%$ ) but Raman measurements were not made at the exact location of the highest concentration. This $\mathrm{Ca}$ and $\mathrm{Na}$ enrichment is very likely due to a very fine mixture of akimotoite together with another phase such as Ca-perovskite glass formed by amorphization of metastable $\mathrm{CaSiO}_{3}$ perovskite upon quenching.

The last type of setting is observed in another grain entrained in the shock melt vein (Fig. 1d). Akimotoite tablets are intermingled with an amorphous phase. Raman measurements show that low brightness areas observed in BSE imaging correspond to a pyroxene glass (broad band at $670 \mathrm{~cm}^{-1}$ ) while the high brightness parts have the akimotoite Raman spectrum. Akimotoite platelets are slightly richer in $\mathrm{Na}\left(\mathrm{Na}_{2} \mathrm{O}=1.03\right)$ and $\mathrm{Ca}(\mathrm{CaO}=0.71)$ compared to the pyroxene glass $\left(\mathrm{Na}_{2} \mathrm{O}=0.75\right.$ and $\left.\mathrm{CaO}=0.65\right)$.

\section{Discussion}

The present observations illustrate more diverse akimotoite occurrences than previously reported (Tomioka and Fujino $[27,28]$ and Sharp et al. [24]). Sharp et al. reported that akimotoite is often associated with ringwoodite and a glassy phase of $(\mathrm{Mg}, \mathrm{Fe}) \mathrm{SiO}_{3}$ composition, the latter possibly being former $(\mathrm{Mg}, \mathrm{Fe}) \mathrm{SiO}_{3}$ perovskite amorphized during the pressure decrease [24]. In the Tenham meteorite, Tomioka and Fujino [27] presented evidence for crystalline perovskite and akimotoite formed from pyroxene through a solid-state transformation. Sharp et al. [24] encountered akimotoite in a different setting whose texture is strongly suggestive that the akimotoite-perovskite intergrowths crystallized from a high-pressure and high-temperature melt. Sharp et al. [24] and Tomioka and Fujino [27] may have encountered two different petrographic settings that were formed by crystallization from the chondritic liquid and by solid-state transformation, respectively. The akimotoite grains analyzed by [27] contain slightly higher amounts of $\mathrm{Na}$ and Ca than those reported for akimotoite synthesized in high-pressure and high-temperature experiments on peridotites compositions [17]. We should however remark that they lie within the uncertainties linked to the EDX techniques used for chemical characterization in these studies.

Here, we observed three different settings for the enstatite to akimotoite conversion and propose three different formation mechanisms. The first one is a solid-state transformation to massive polycrystalline akimotoite occurring near the interface of a solid grain (Fig 1 b) or at vein wall (Fig 1c) of the shock melt vein. The second mechanism involved is an intracrystalline solid-state transformation inside one of the grains (Fig 3c). The third mechanism could be a back transformation of a perovskite/akimotoite phase to a pyroxene glass mixed with akimotoite (Fig 1d) or a "lowtemperature" transformation of enstatite to akimotoite and diaplectic glass.

Among the observed transformation settings of pyroxene to akimotoite, the two first transformation mechanisms have already been documented experimentally for olivine polymorphic transitions $[8,12,16,9,10]$. The first mechanism 
would be similar to the "granular" type reported by [27] and was documented for olivine-ringwoodite in the same meteorite by Xie et al. [31]. The second mechanism shares similarity to the "columnar-type" akimotoite reported by [27] and observed for olivine-ringwoodite in the Tenham chondrite by Beck et al. [3]. However, we have no indication that lamellar akimotoite is monocrystalline and could therefore be analogous to the "columnar akimotoite" of [27]. Furthermore, Miyahara et al. [14] reported that ringwoodite lamellae in the Peace River Meteorite were formed through frictional melting and crystallization from this melt at high pressure. Akimotoite lamellae could have originated through a similar mechanism.

To explain the occurrence of either the massive polycrystalline transformation or the intracrystalline type, we propose two different alternative models that can be tested for the documented pyroxene. Both models involve a temperature gradient inside the grain (temperature is likely higher in the precursor of the polycrystalline akimotoite part away from the vein wall than in the enstatite closer to the vein wall).

In the first model, the gradient is assumed to favor different transformation mechanisms by analogy with the olivineringwoodite transformation. For the olivine system, the activation energy of polycrystalline growth kinetics is higher than for intracrystalline growth, showing that the polycrystalline transformation is more difficult to start. On the other hand, kinetics are faster for the polycrystalline transformation than for the intracrystalline transformation $[8,12,16,9$, 10]. If we hypothesize a similar behavior for the olivine-ringwoodite and enstatite-akimotoite systems, the higher temperature in the zone of polycrystalline akimotoite would have allowed both transformations to occur simultaneously. Kinetics of the polycrystalline phase being faster, it would have rapidly overridden the intracrystalline mechanism provided no friction melting took place. The lower temperature close to the vein wall would have allowed only the intracrystalline transformation. Nucleation kinetics being highly dependent on the density of nucleation sites, which are not known in the present sample, we cannot discuss about the nucleation kinetics.

In a second model (Fig 5b), a polycrystalline transformation is initiated at the boundary with the shock melt vein and the transformation front progresses inside the enstatite grain. The interface of the akimotoite-enstatite transformation acts afterwards as nucleation sites for intracrystalline growth. The transition from polycrystalline to intracrystalline mechanisms can be a result of the temperature gradient descending into the enstatite grain because of temperature decrease during crystallization of the SMV from the wall with solid meteorite. This model is supported by the SEM observations, which show that major lamellae are radiating away from the enstatite-akimotoite boundary into the interior of enstatite.

In the case of the akimotoite formed at the vein wall (Fig 1c), no akimotoite lamellae is observed, only a polycrystaline stripe. This may be due to a steeper temperature gradient across the transformation zone.

The chemical composition of akimotoite obtained from microprobe or EDX on SEM is intriguing especially because of significant $\mathrm{Ca}$ and $\mathrm{Na}$ contents, and is related to the occurrence of polycrystalline patches. The higher concentrations of calcium, sodium and aluminum in akimotoite compared to enstatite suggest diffusion of these elements from the shock melt vein into the akimotoite. Inherited zoning from the former magmatic history of the enstatite precursor is unlikely because of the high $\mathrm{Na}$ and $\mathrm{Al}$ contents. Enrichment from the shock melt vein is supported by the Ca distribution map, which shows a relative depletion of calcium in the SMV adjacent to the akimotoite grain compared to the SMV adjacent to enstatite. The coincidence of the front of the $\mathrm{Na}, \mathrm{Al}$ or $\mathrm{Ca}$ diffusion and the front of the polycrystalline akimotoite formation could also suggest melt injection in the porosity linked with the volume decrease during the enstatite to akimotoite transformation. A similar enrichment of $\mathrm{Na}(\mathrm{NaO}=1.72 \%), \mathrm{Al}\left(\mathrm{Al}_{2} \mathrm{O}_{3}=4.14 \%\right)$ and $\mathrm{Ca}(\mathrm{CaO}=2.85 \%)$ is observed in the second occurrence of polycrystalline akimotoite at the vein wall. These chemical differences show that significant amounts of calcium and sodium can be stored in akimotoite, which was not expected from previous experiments [17]. Alternatively, in the case of the grain inside the SMV, the difference in chemical composition could also be explained by the presence of pigeonite revealed by X-ray diffraction, and having a higher concentration in $\mathrm{Ca}$, $\mathrm{Na}$ and $\mathrm{Al}$ than enstatite.

The third mechanism of akimotoite transformation is encountered in the grain entrained in the shock melt vein (Fig 1d). It consists of the mixture of interwoven akimotoite and pyroxene glass. Because of the grain shape and the morphology of the akimotoite platelets, several mechanisms can be proposed for the pyroxene glass formation: 1) it is a backtransformation product by complete amorphization of a former perovskite $[5,30]$ mixed with akimotoite or partial amorphization of akimotoite [21] ; 2) partial transformation to high-pressure glass and akimotoite at relatively "low" temperatures; 3) crystallization of akimotoite or akimotoite-perovskite assemblage from a melt, with subsequent amorphization of perovskite. The latter mechanism would involve large chemical fractionation of elements such as Al, $\mathrm{Ca}, \mathrm{Na}$, which is not observed; it is thus very unlikely. The two other mechanisms occur at solid state and involve limited diffusion, thereby explaining the similar composition of glass and akimotoite. The observation of a perovskite diffraction by TEM prior to amorphization in a similar texture and composition by Tomioka and Fujino [27] supports the first mechanism. In the opposite, Sharp et al. [24] observed a glass with akimotoite of different compositions, supporting crystallization from a melt.

\section{Conclusion}

By analogy with the olivine-ringwoodite transformation, two different mechanisms are proposed for the enstatiteakimotoite system. One mechanism involves growth of a polycrystalline akimotoite from a grain boundary with shock melt vein or at the vein wall. The second mechanism is involving an isochemical intracrystalline growth of akimotoite at the expense of enstatite. The first mechanism is not entirely isochemical, and is associated with a diffusive 
redistribution of calcium, aluminum and sodium from the adjacent chondritic shock melt vein inside the polycrystalline akimotoite. It remains to be determined whether akimotoite might stock up to more than $2 \%$ of $\mathrm{Ca}$ and $\mathrm{Na}$, or is systematically associated with $\mathrm{Na}$, Ca-rich phase such as pigeonite or melt. Finally, the occurrence of akimotoite together with pyroxene glass of similar composition could be interpreted as a previous mixture of akimotoite and perovskite which would have back transformed to amorphous pyroxene by a moderate post-shock residual temperature $[27,28]$.

\section{Acknowledgments}

The authors warmly thank Gilles Montagnac for his technical assistance on the Raman mapping. We also thank Rémi Tucoulou from ID22 and Michael Hanfland from ID09 for help during X-ray diffraction experiments at ESRF. MATEIS Lab is also thanked for providing access to the SEM facility. This project was supported by ANR project ECSS. We acknowledge the constructive reviews by an anonymous reviewer and N. Tomioka that helped in improving the manuscript.

\section{References}

[1] : R.J. Angel, D.A. Hugh-Jones, Equations of state and thermodynamic properties of enstatite pyroxenes, J. Geophys. Res. 99 (B10) (1994) 19777-19783 Scopus

[2] : W.H. Baur, Computer-simulated crystal structures of observed and hypothetical Mg2SiO4 polymorphs of low and high density, Am. Min. 57 (1972) 709-731

[3] : P. Beck, P. Gillet, A. El Goresy, S. Mostefaoui, Timescales of shock processes in chondritic and Martian meteorites, Nature 435 (2005) 1071-1074 $\underline{\text { Scopus. }}$

[4] : R.A. Binns, R.J. Davis, S.J.B. Reed, Ringwoodite, natural (Mg,Fe)2SiO4 spinel in the Tenham meteorite, Nature 221 (1969) 943-944 $\underline{\text { Scopus. }}$

[5] D.J. Durben and G.H. Wolf, High-temperature behavior of metastable $\mathrm{MgSiO}_{3}$ perovskite: a Raman spectroscopic study, Am. Mineral. 77 (1992), pp. 890-893 $\underline{\text { Scopus. }}$

[6] : A. Hogrefe, D.C. Rubie, T.G. Sharp, F. Seifert, Metastability of enstatite in deep subducting lithosphere, Nature 372 (1994) 351-353 Scopus.

[7] : H. Horiuchi, M. Hirano, E. Ito, Y. Matsui, MgSiO3 (ilmenite-type): Single crystal X-ray diffraction study, Am. Min. 67 (1982) 788-793

[8] : L. Kerschhofer, C. Dupas, M. Liu, T.G. Sharp, W.B. Durham, D.C. Rubie, Polymorphic transformations between olivine, wadsleyite and ringwoodite: mechanisms of intracrystalline nucleation and the role of elastic strain, Mineral. Mag. 62 (5) (1998) 617-638 Scopus.

[9] : L. Kerschhofer, D.C. Rubie, T.G. Sharp, J.D.C. McConnell, C. Dupas-Bruzek, Kinetics of intracrystalline olivine-ringwoodite transformation, Phys. Earth Planet. Inter. 121 (2000) 5976.

[10] : T. Kubo, E. Ohtani, K. Funakoshi, Nucleation and growth kinetics of the $\alpha-\beta$ transformation in $\mathrm{Mg} 2 \mathrm{SiO} 4$ determined by in situ synchrotron powder X-ray diffraction, Am. Min. 89 (2004) 285-293 $\underline{\text { Scopus }}$

[11] : F. Langenhorst, J. Pascal, JC. Doukhan, Thermal and shock metamorphism of the Tenham chondrite: A TEM examination, Geochim. Cosmochim. Acta 59 (1995)1835-1845 Scopus.

[12] : M. Liu, L. Kerschhofer, J. L. Mosenfelder, D. C. Rubie, The effect of strain energy on growth rates during the olivine-spinel transformation and implications for olivine metastability in subducting slabs, J. Geophys. Res. 103 (B10) (1998) 23897-23910 $\underline{\text { Scopus }}$

[13] : B. Mason, J Nelen, J.S. White Jr, Olivine-Garnet Transformation in a Meteorite, Science 160 (3823) (1968) 66-67 $\underline{\text { Scopus }}$ 
[14] : M. Miyahara, A. El Goresy, E. Ohtani, T. Nagase, M. Nishijima, Z. Vashaei, T. Ferroir, P. Gillet, L. Dubrovinsky, A. Simionovici, Evidence for fractional crystallization of wadsleyite and ringwoodite from olivine melts in chondrules entrained in shock-melt veins, PNAS 105 (25) (2008) (8542-8547).

[15] : J.L. Mosenfelder, J.A.D. Connolly, D.C. Rubie, M. Liu, Strength of $(\mathrm{Mg}, \mathrm{Fe})_{2} \mathrm{SiO}_{4}$ wadsleyite determined by relaxation of transformation stress, Phys. Earth Planet. Inter. 120 (1-2) (2000) 63-78 Scopus.

[16] : J.L. Mosenfelder, F.C. Marton, C.R. Ross, L. Kerschhofer, D.C. Rubie, Experimental constraints on the depth of olivine metastability in subducting lithosphere, Phys. Earth Planet. Inter. 127 (1-4) (2001) 165-180 Scopus.

[17] : N. Nishiyama, T. Yagi, Phase relation and mineral chemistry in pyrolite to $2200^{\circ} \mathrm{C}$ under the lower mantle pressures and implications for dynamics of mantle plumes, J. Geophys. Res. 108 (B5) (2003) 2255-2267

[18] : E. Ohtani, Y. Kimura, M. Kimura, T. Takata, T. Kondo, T. Kubo, Formation of highpressure minerals in shocked L6 chondrite Yamato 791384: constraints on shock conditions and parent body size, Earth Planet. Sci. Lett. 227 (3-4) (2004) 505-515.

[19] : G.D. Price, A. Putnis, S.O. Agrell, Electron petrography of shock-produced veins in the Tenham chondrite, Contributions to Mineralogy and Petrology 71 (1979) 211-218 Scopus

[20] : A. Putnis, G.D. Price, High-pressure (Mg, Fe)2SiO4 phases in the Tenham chondritic meteorite, Nature 280 (1979) 217-218 $\underline{\text { Scopus. }}$

[21] : B. Reynard, D.C. Rubie, High-pressure, hight-temperature Raman spectroscopic study of ilmenite-type MgSiO3, Am. Min. 81 (1996) 1092-1096 Scopus

[22] : B. Reynard, A. Kubo, M. Akaogi, Raman spectra of ilmenite-type phases on the Mg4Si4O12Mg3Al2Si3O12 join, Eur. J. Mineral. 14 (2002) 745-747 $\underline{\text { Scopus }}$

[23] : N.L. Ross, P. McMillan, The Raman spectrum of MgSiO3 ilmenite, Am. Min. 69 (1984) 719721 Scopus

[24] : T.G. Sharp, C.M. Lingemann, C. Dupas, D. Stöffler, Natural occurrence of MgSiO3-ilmenite and evidence for MgSiO3- perovskite in a shocked L chondrite, Science 277 (1997) 352-355.

[25] : J.V. Smith, B. Mason, Pyroxene-garnet transformation in Coorara meteorite, Science 168 (3933) (1970) 832-833.

[26] : D. Stöffler, K. Keil, E.R.D. Scott, Shock metamorphism of ordinary chondrites, Geochim. Cosmochim. Acta 55 (1991)3845-3867 Scopus.

[27] : N. Tomioka, K. Fujino, Natural (Mg,Fe)SiO3-ilmenite and -perovskite in the Tenham meteorite, Science 277 (1997) 1084-1086 Scopus.

[28] : N. Tomioka, K. Fujino, Akimotoite, $(\mathrm{Mg}, \mathrm{Fe}) \mathrm{SiO} 3$, a new silicate mineral of the ilmenite group in the Tenham chondrite, Am. Min. 84 (1999) 267-271 Scopus.

[29] N. Tomioka, A model for the shear mechanism in the enstatite-akimotoite phase transition, Journal of Mineralogical and Petrological Science 102 (2007) 226-232 Scopus

[30] : Y. Wang, F. Guyot, R.C. Liebermann, Electron Microscopy of (Mg, Fe)SiO3 Perovskite: Evidence for Structural Phase Transitions and Implications for the Lower Mantle, J. Geophys. Res. 97 (B9) (1992) 12327-12347 $\underline{\text { Scopus }}$

[31] : Z. Xie, T.G. Sharp, P.S. DeCarli, High pressure phases in a shock-induced melt vein of Tenham L6 Chondrite: constraints on shock pressure and duration, Geochim. Cosmochim. Acta 70 (2006) 504-515 $\underline{\text { Scopus. }}$ 


\section{Figures caption}

\section{Table 1}

Chemical composition of akimotoite, enstatite and pyroxene glass obtained by electron microprobe in the Tenham Chondrite.

* data from Tomioka et and Fujino. (1997)

** data from Sharp et al.(1997)

Table 2

Observed and calculated d-values of akimotoite using a wavelength of $0.412 \AA$.

Fig.1 Back scattered electron (BSE) images of the section of the Tenham chondrite. Upper left (a) is the global view of the section with the locations of the investigated akimotoite. Bottom square shows the location of (b), center square the location of (c) and the top one shows (d). (b) grain located inside the shock melt vein which displays both polycrystalline and intracrystalline transformation. (c) akimotoite grain at the vein wall partially resorbed by the melt. (d) akimotoite tablets interwoven with pyroxene glass.

$\mathrm{Ak}=$ akimotoite

Fig.2 Raman spectra of akimotoite (upper spectrum) and enstatite (lower spectrum) collected on the first grain entrained in the shock melt vein. The $800 \mathrm{~cm}^{-1}$ peak in the pyroxene spectrum comes from minor amount of akimotoite.

Fig.3 Back-scattered electron (BSE) image of the first studied grain entrained in the shock melt vein (a) AkP : Polycrystalline akimotoite, AkI : Intracrystalline akimotoite, En : Enstatite. (b) High magnification BSE picture of the akimotoite lamellae which show three different types of orientations. (c) Raman map of the grain located inside the shock melt vein. Colormap is Red for akimotoite, green for enstatite, blue for sulfides from the shock melt vein and purple for ringwoodite. This map is well correlated with both optical and BSE observation. (d) Chemical map of the same grain located inside the shock melt vein determined by electron microprobe. Color scale represents the counts per second for Calcium $(\mathrm{CaK} \alpha)$ in linear scale. Akimotoite part (Ak) is enriched in calcium compared to enstatite (En). Akimotoite lamellae cannot be distinguished since their chemical composition is identical to the enstatite host. The melt vein near the akimotoite part is depleted in calcium compared to SMV near the enstatite, suggestive of calcium diffusion in akimotoite from the SMV.

Fig. 4a Integrated X-ray diffraction spectra of the previous image plate. Major peaks can be assigned to akimotoite and ringwoodite while minor peaks correspond to enstatite. Wavelength was $0.412 \AA$

b Integrated pattern of the imaging plate showing that akimotoite is mixed with pigeonite. Iron and ringwoodite diffraction lines are also present in the spectra. Wavelength was $1.033 \AA$

Fig 5. The two different models for the growth of polycrystalline and intracrystalline akimotoite. The first one (a) is driven by the temperature gradient inside the grain while the second one (b) involves cooling of the shock melt vein. 
Table 1

Click here to download Table: Table1.xls

Feuille1

\begin{tabular}{|c|c|c|c|c|c|c|c|c|c|c|}
\hline $\begin{array}{l}\text { Oxide } \\
\text { (wt } \%)\end{array}$ & $\begin{array}{c}\text { Aki grain } \\
\text { (polycrystalline) }\end{array}$ & $\begin{array}{c}\text { Enstatite and } \\
\text { intracrystalline } \\
\text { aki in grain }\end{array}$ & $\begin{array}{c}\text { Aki } \\
\text { (vein wall) }\end{array}$ & $\begin{array}{c}\text { Px } \\
\text { glass }\end{array}$ & $\begin{array}{c}\text { Aki } \\
\text { (flakes) }\end{array}$ & $\mathbf{A k i}^{*}$ & Cen* & $\mathbf{P v}^{*}$ & $\mathbf{A} \mathbf{k i}$ ** & $P v$ ** \\
\hline $\mathrm{Na} 2 \mathrm{O}$ & 1.73 & 0.18 & 1.72 & 0.75 & 1.03 & 0.67 & 0.01 & 0.81 & 0.42 & 2.02 \\
\hline $\mathrm{K}_{2} \mathrm{O}$ & 0.11 & 0.02 & 0.14 & NM & NM & NM & NM & NM & NM & NM \\
\hline $\mathrm{FeO}$ & 13.4 & 14.38 & 14.85 & 14.21 & 14.12 & 13.54 & 13.51 & 14.41 & 3.72 & 13.16 \\
\hline $\mathrm{SiO}_{2}$ & 54.2 & 55.27 & 51.17 & 54.12 & 54.17 & 56.35 & 55.74 & 55.14 & 55.54 & 49.14 \\
\hline MgO & 22.95 & 28.91 & 23.12 & 29.47 & 28.95 & 28.39 & 29.33 & 28.58 & 35.34 & 26.24 \\
\hline $\mathrm{CaO}$ & 1.74 & 0.71 & 2.85 & 0.65 & 0.71 & 0.38 & 0.78 & 0.12 & NM & 3.95 \\
\hline MnO & 0.4 & 0.45 & 0.35 & 0.52 & 0.51 & 0.28 & 0.51 & 0.19 & NM & 0.81 \\
\hline $\mathrm{Al}_{2} \mathrm{O}_{3}$ & 4.18 & 0.25 & 4.14 & 0.17 & 0.15 & 0.07 & 0.15 & 0.16 & 4.44 & 4.69 \\
\hline $\mathrm{Cr}_{2} \mathrm{O}_{3}$ & 0.23 & 0.06 & 0.28 & 0.19 & 0.23 & 0.16 & 0.16 & 0.18 & 0.5 & tr \\
\hline $\mathrm{TiO}_{2}$ & 0.13 & 0.14 & 0.14 & 0.18 & 0.2 & 0.17 & 0.15 & 0.42 & NM & NM \\
\hline TOTAL & 99.09 & 100.39 & 98.79 & 100.26 & 100.07 & 100.01 & 100.34 & 99.76 & 99.96 & 100.01 \\
\hline
\end{tabular}


Table 2

Click here to download Table: Table2.xls

Feuille1

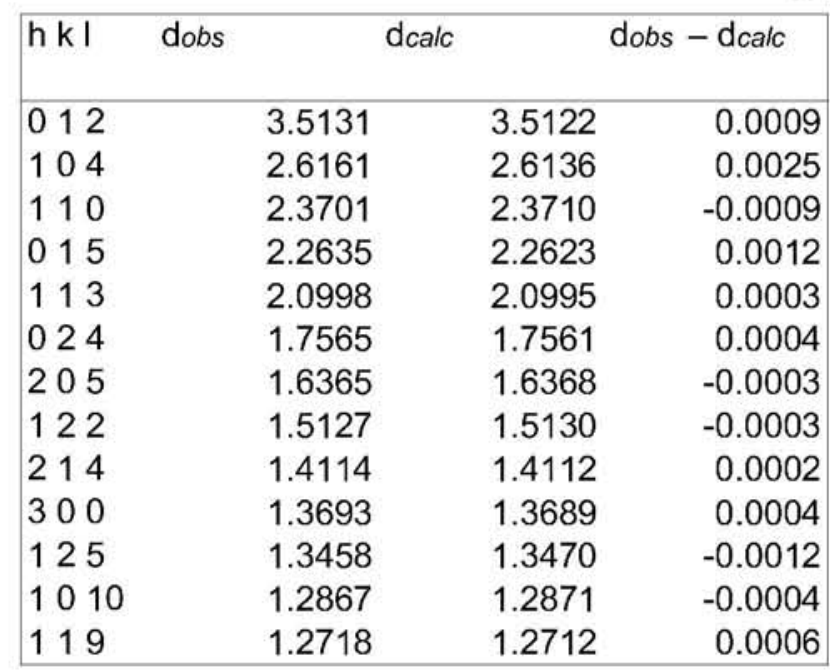

Page 1 

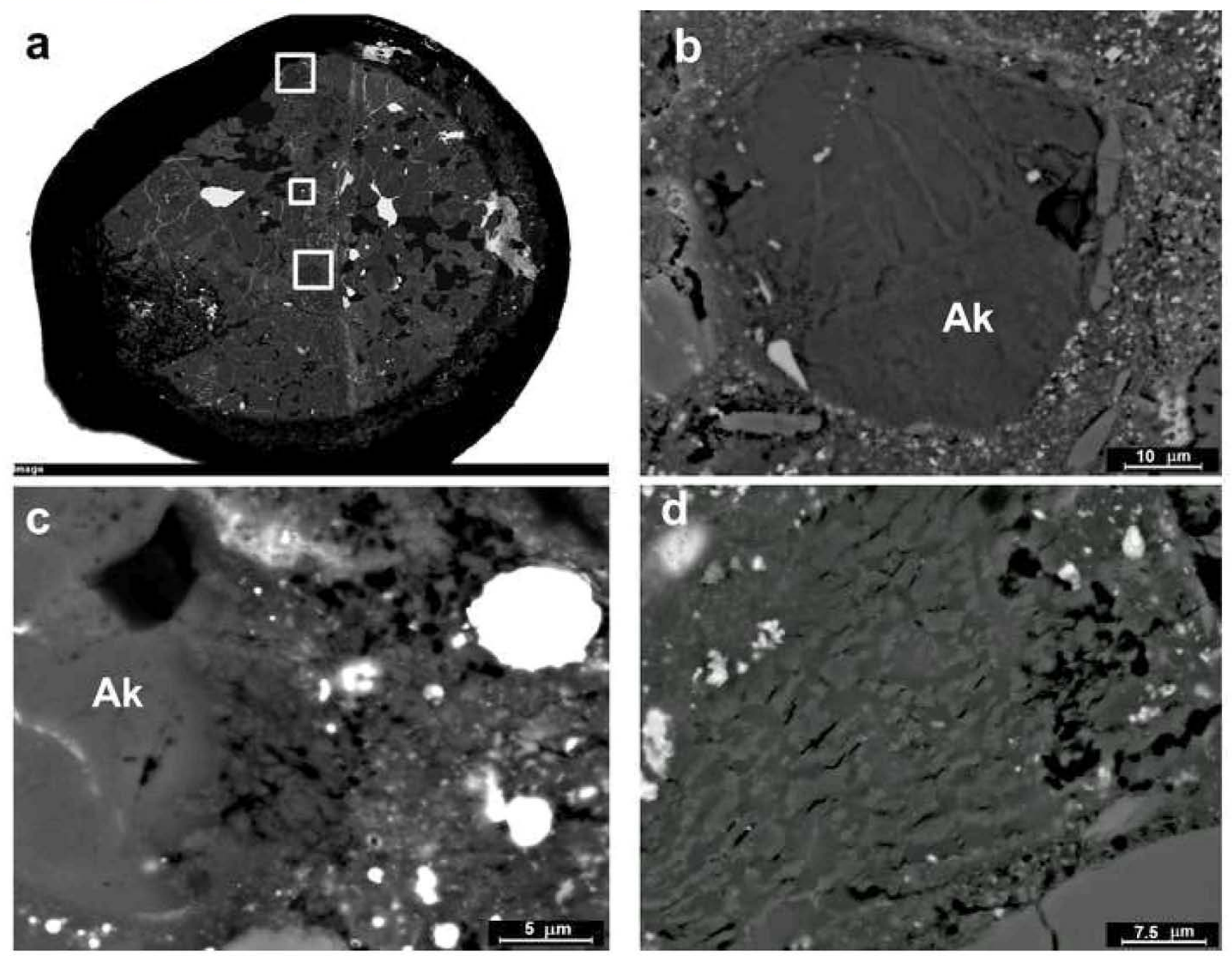


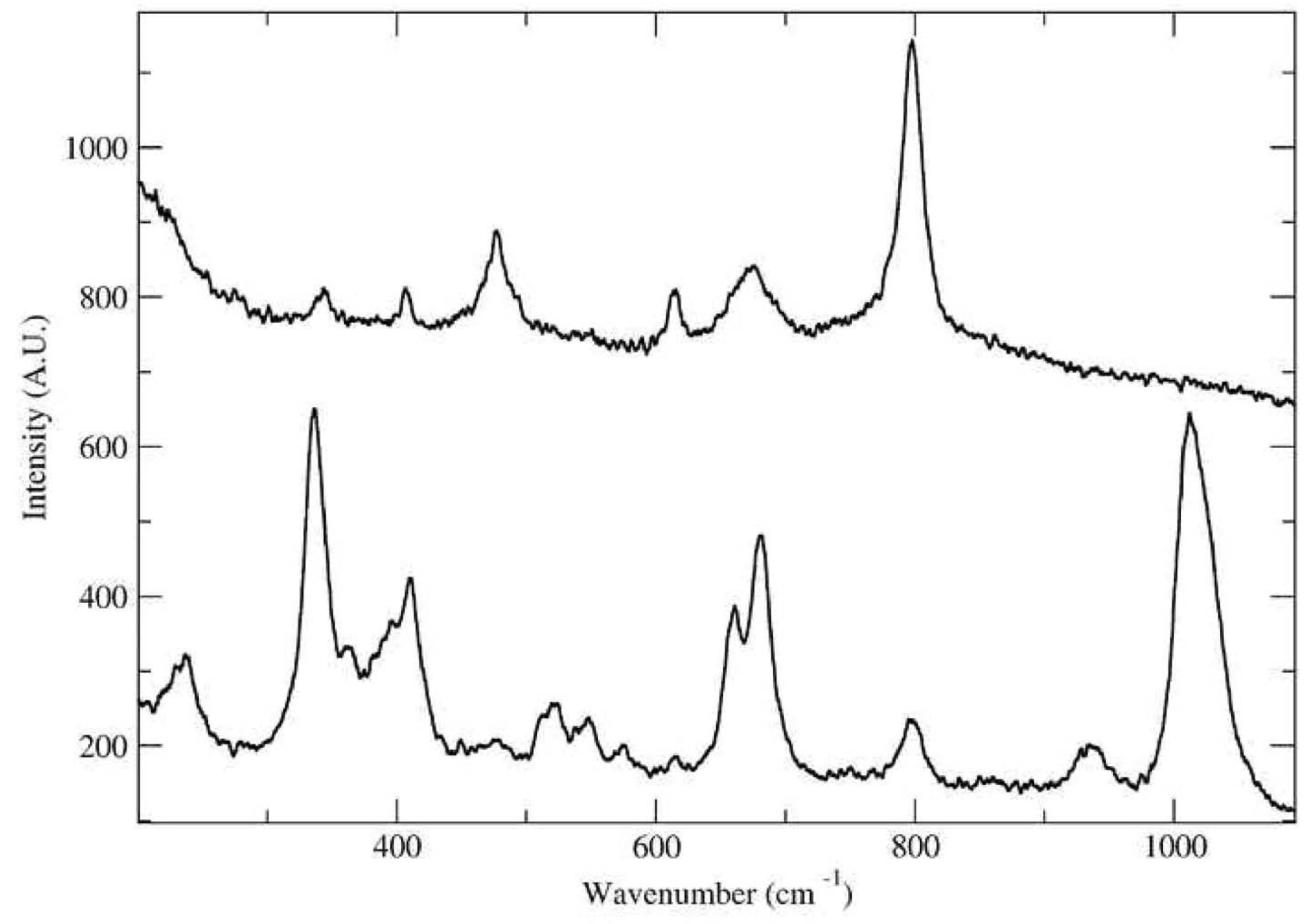



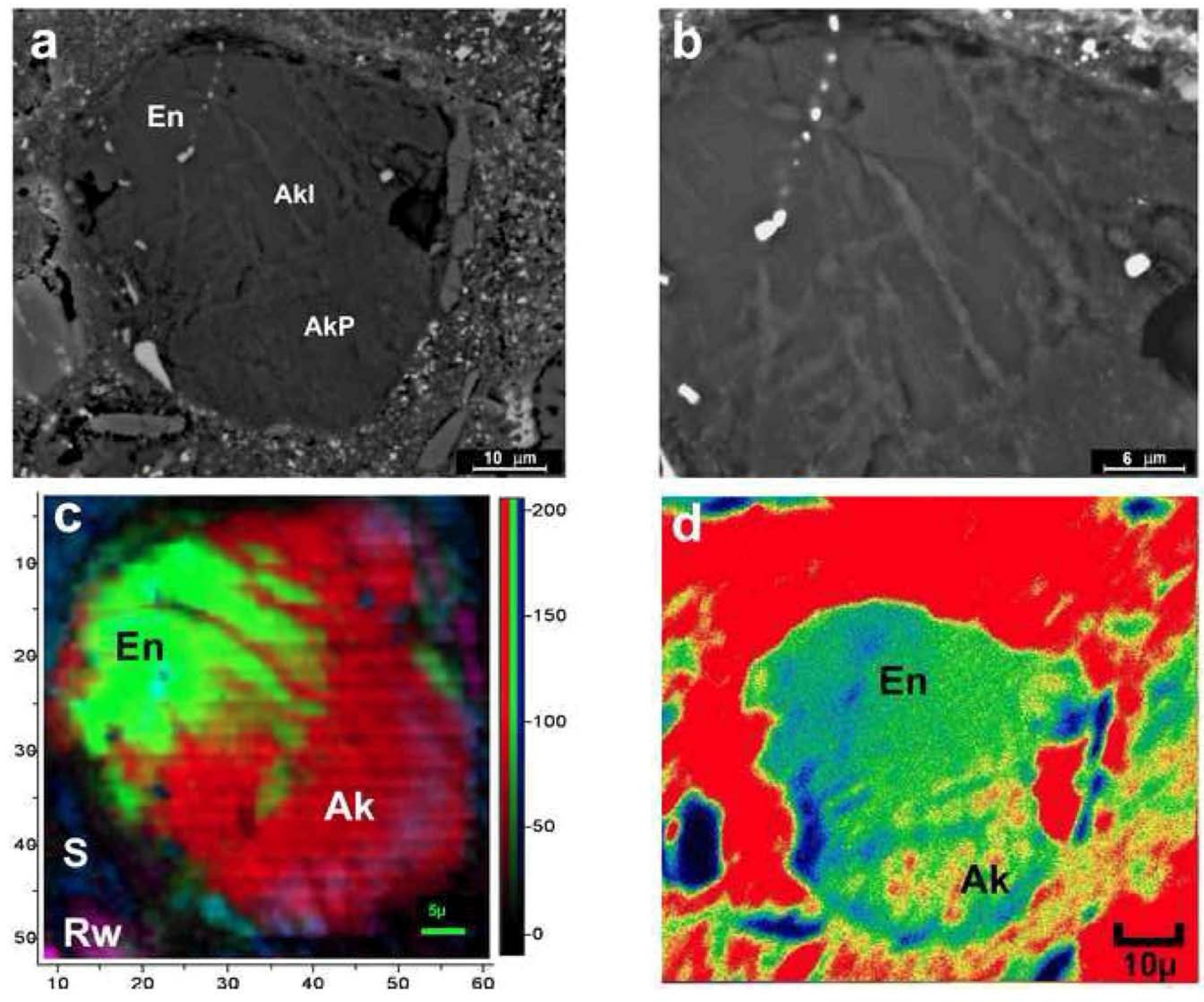

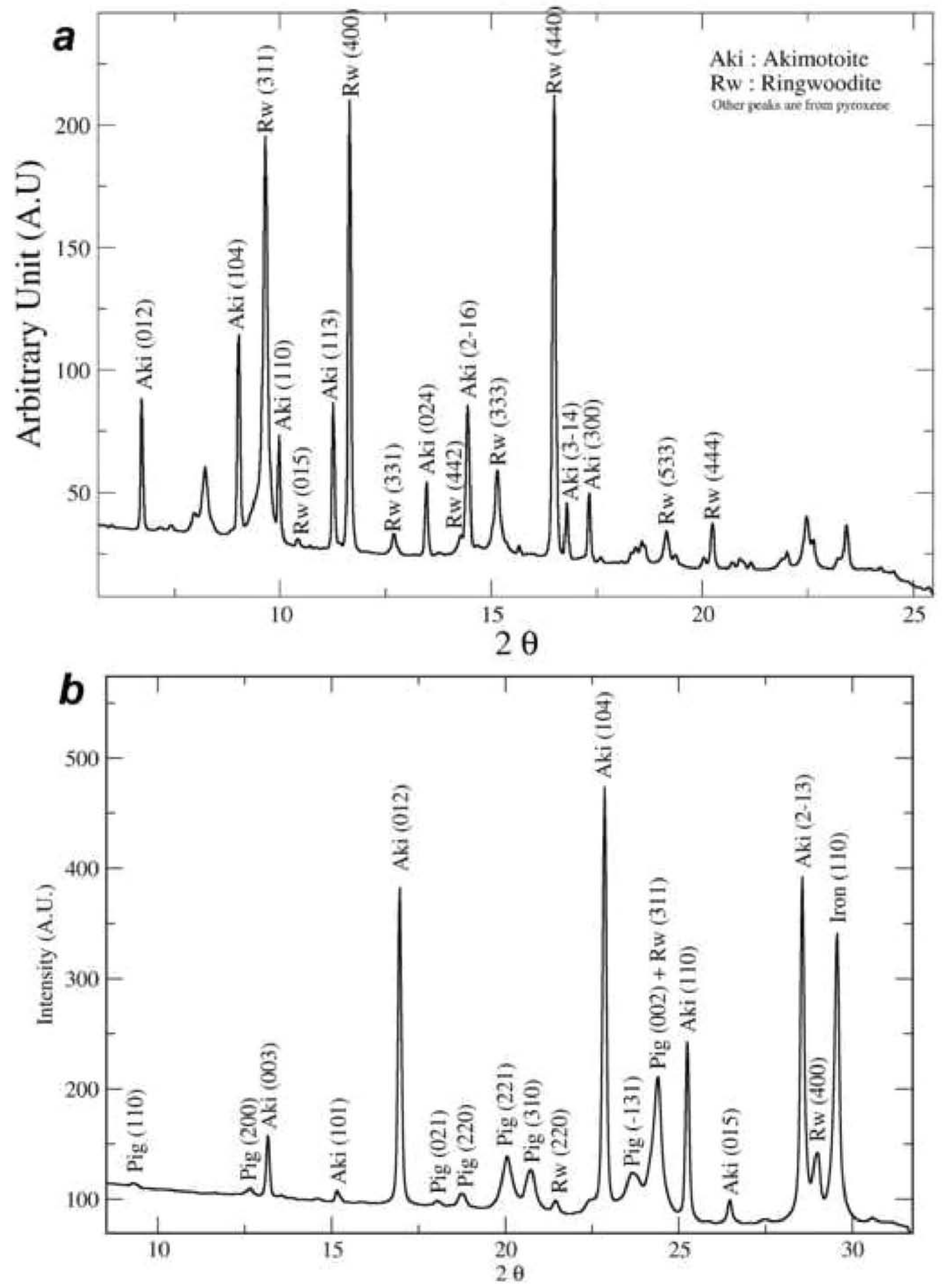

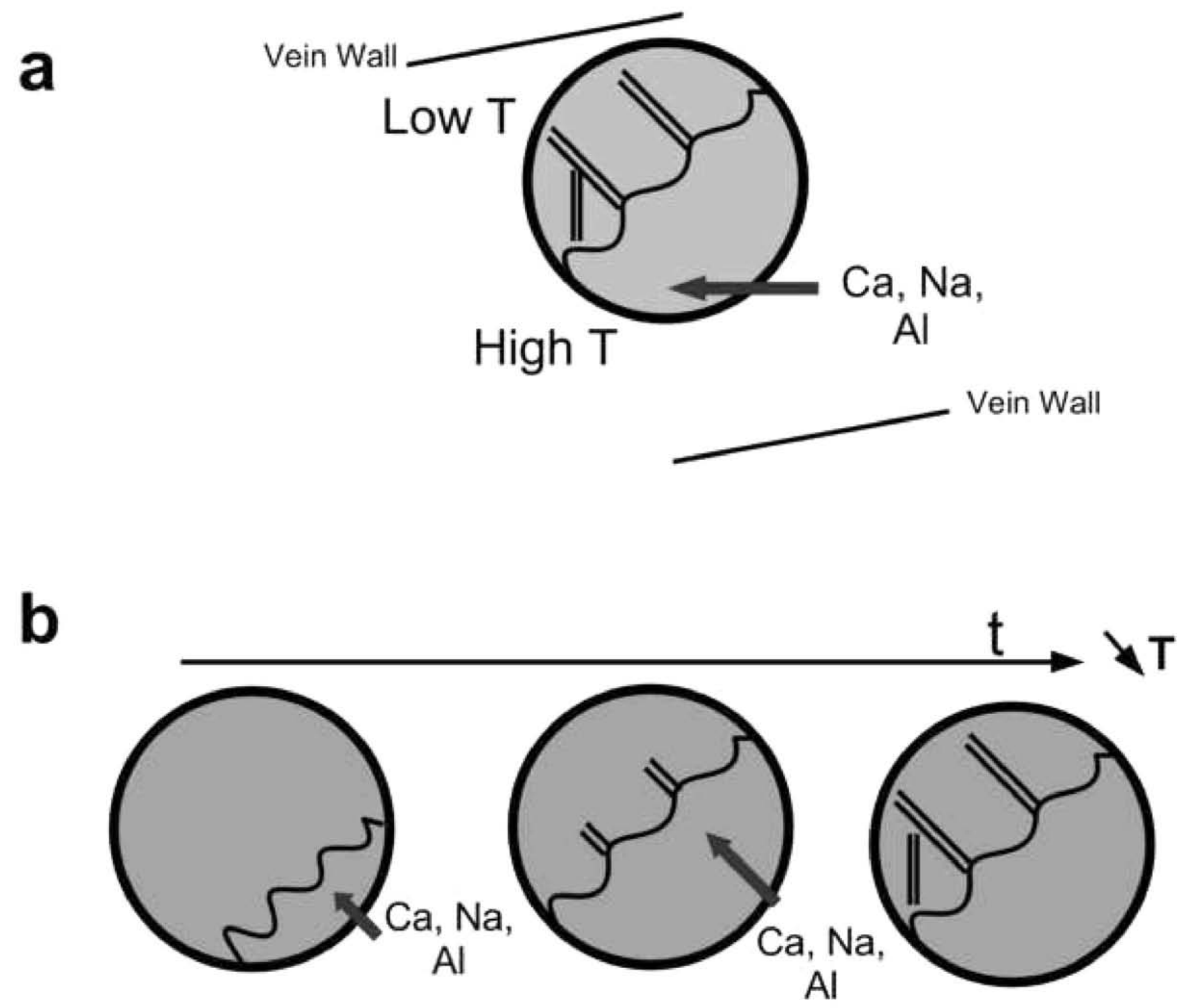